\title{
McMYB4 improves temperature adaptation by regulating phenylpropanoid metabolism and hormone signaling in apple
}

\author{
Suxiao Hao ${ }^{1,2,3,4,5}$, Yanfen Lu ${ }^{1,3,4}$, Zhen Peng ${ }^{1,3,4}$, Enying Wang ${ }^{3,4}$, Linke Chao ${ }^{3,4}$, Silin Zhong ${ }^{1,6 凶}$ and
} Yuncong Yao (1) $1,3,4 \times$

\begin{abstract}
Temperature changes affect apple development and production. Phenylpropanoid metabolism and hormone signaling play a crucial role in regulating apple growth and development in response to temperature changes. Here, we found that MCMYB4 is induced by treatment at $28^{\circ} \mathrm{C}$ and $18^{\circ} \mathrm{C}$, and MCMYB4 overexpression results in flavonol and lignin accumulation in apple leaves. Yeast one-hybrid $(\mathrm{Y} 1 \mathrm{H})$ assays and electrophoretic mobility shift assays (EMSAs) further revealed that MCMYB4 targets the promoters of the flavonol biosynthesis genes CHS and FLS and the lignin biosynthesis genes $C A D$ and $F 5 H$. MCMYB4 expression resulted in higher levels of flavonol and lignin biosynthesis in apple during growth at $28^{\circ} \mathrm{C}$ and $18{ }^{\circ} \mathrm{C}$ than during growth at $23^{\circ} \mathrm{C}$. At $28{ }^{\circ} \mathrm{C}$ and $18{ }^{\circ} \mathrm{C}$, McMYB4 also binds to the AUXIARF and BRI/BIN promoters to activate gene expression, resulting in acceleration of the auxin and brassinolide signaling pathways. Taken together, our results demonstrate that MCMYB4 promotes flavonol biosynthesis and brassinolide signaling, which decreases ROS contents to improve plant resistance and promotes lignin biosynthesis and auxin signaling to regulate plant growth. This study suggests that MCMYB4 participates in the abiotic resistance and growth of apple in response to temperature changes by regulating phenylpropanoid metabolism and hormone signaling.
\end{abstract}

\section{Introduction}

As an important environmental factor, temperature often impacts plant growth, development and productivity during the growing season ${ }^{1}$. Therefore, increasing attention has been directed toward the regulation of plants in response to temperature changes ${ }^{2}$. Plants are believed to activate protective mechanisms during their adaptation to temperature changes by balancing resistance and growth. For example, under high-temperature conditions, SLG1 (Slender Guy 1) could encode cytosolic tRNA 2-thiolation

Correspondence: Silin Zhong (silin.zhong@gmail.com) or

Yuncong Yao (yaoyc_20@126.com)

Beijing Advanced Innovation Center for Tree Breeding by Molecular Design, Beijing University of Agriculture, Beijing 102206, China

${ }^{2}$ Beijing Bei Nong Enterprise Management Co. Ltd, Beijing 102206, China

Full list of author information is available at the end of the article

These authors contributed equally: Suxiao Hao, Yanfen Lu, Zhen Peng and

Enying Wang protein 2 (RCTU2) by regulating its promoter and coding regions, which led to an increase in thiolated tRNA and enhanced resistance and thus improved rice yields ${ }^{3}$. In Arabidopsis thaliana, the photosystem II (PSII)-associated protein PSB27 downregulated PSII electron transport under low-temperature conditions to affect plant photosynthesis and growth ${ }^{4}$.

Apple (Malus domestica) is frequently exposed to challenges from various environmental factors. Temperature has a serious impact on growth and development processes, which affects apple yields ${ }^{5}$. At a low temperature $\left(15^{\circ} \mathrm{C}\right)$, McMYB10 bound to the promoter of flavonoid 3'-hydroxylase $\left(M c F 3^{\prime} H\right)$ and promoted its expression to enhance anthocyanin accumulation in crabapple leaves and calli, which promoted apple coloration ${ }^{6}$. In apple, MdERF1BMdCIbHLH1 upregulated the expression of the ethylene biosynthesis genes MdACO1 and MdERF3 and promoted

\section{(c) The Author(s) 2021}

(c) (i) Open Access This article is licensed under a Creative Commons Attribution 4.0 International License, which permits use, sharing, adaptation, distribution and reproduction cc) in any medium or format, as long as you give appropriate credit to the original author(s) and the source, provide a link to the Creative Commons license, and indicate if changes were made. The images or other third party material in this article are included in the article's Creative Commons license, unless indicated otherwise in a credit line to the material. If material is not included in the article's Creative Commons license and your intended use is not permitted by statutory regulation or exceeds the permitted use, you will need to obtain permission directly from the copyright holder. To view a copy of this license, visit http://creativecommons.org/licenses/by/4.0/. 
ethylene production, which positively modulated the response of apple to $4{ }^{\circ} \mathrm{C}^{7}$. Therefore, substance metabolism and hormone signaling in apple plants play a crucial role in their response to temperature changes.

Phenylpropanoid metabolism is an important secondary metabolic process in the plant kingdom ${ }^{8}$. Flavonol, a key component in this metabolic pathway, can increase plant resistance and regulate plant growth. For instance, the flavonol contents in leaves of a hybrid Arabidopsis line are higher than those in leaves of Columbia plants, and this difference is accompanied by greater accumulation of soluble sugars and a higher proline content after a 14-day low-temperature treatment, which increases plant freezing tolerance 9 . Moreover, the flavonol contents in Arabidopsis thaliana were also correlated with a shortened plant stature. The flavonoid 3-O-glucosyltransferase mutant ugt78d2 regulated the flavonol glycoside pattern and reduced polar auxin transport (PAT) in shoots, which resulted in a dwarf-type stature ${ }^{10}$. Lignin, as a complex phenolic polymer, can affect the cell wall and vascular structure and thereby regulate plant growth and resistance ${ }^{11,12}$. For example, expression of the p-coumaroyl ester 3-hydroxylase gene $C^{\prime} H$ promoted lignin biosynthesis and changed the cell wall structure, thereby regulating plant growth and dwarfing in rice ${ }^{13}$. In maize roots, the fraction of the lignocellulosic complex in the cell wall was significantly increased, which resulted in lignin deposition and root growth promotion ${ }^{14}$. Furthermore, chilling temperatures $\left(8^{\circ} \mathrm{C} / 4^{\circ} \mathrm{C}\right)$ also promoted lignified xylem deposition in cell walls by regulating the transcriptional levels of secondary cell wall (SCW) genes in Eucalyptus gunnii $\times$ Eucalyptus dalrympleana hybrids. The expression levels of most genes involved in lignin biosynthesis were upregulated and contributed to the cold tolerance of the hybrids ${ }^{15}$. These results indicate that flavonol and lignin play an important role in plant growth and resistance. However, their effects on apple in response to temperature changes need further study.

Phytohormone signaling can also regulate plant growth and resistance in response to temperature changes. Under low-temperature conditions, RNA sequencing (RNA-seq) revealed that the $\mathrm{C}$-repeat binding transcription factor (CBF1) promoted the expression of genes involved in auxin, $\mathrm{GA}$, and cytokinin signaling in peach and affected hormone homeostasis and growth ${ }^{16}$. In apple, MdPIF4 could transactivate the MdYUCCA8a promoter, which promoted indole-3-acetic acid (IAA) accumulation and further improved plant height, thus affecting apical dominance and silique malformation under high-temperature conditions ${ }^{17}$. Moreover, high temperatures also promoted the expression of auxin biosynthesis pathway genes (GmYUCCA3, GmYUCCA5 and GmYUCCA7) to increase IAA accumulation and promoted soybean hypocotyl elongation ${ }^{18}$. However, under freezing conditions, overexpression of
BYPASS1-LIKE (B1L) decreased hypocotyl length and fresh weight in Arabidopsis by regulating the BR signaling pathway. Furthermore, the TRANSTHYRETIN-LIKE (TTL) ttl-1 mutant promoted freezing tolerance. Therefore, $B 1 L$ overexpression promoted plant freezing tolerance by interacting with $\mathrm{TTL}^{19}$. BR could also positively regulate the net photosynthetic rate and stomatal conductance under lowtemperature treatment $\left(8^{\circ} \mathrm{C}\right)$, which promoted the growth and development of tung trees ${ }^{20}$. These results suggest that phytohormones have a major impact on the regulation of plant growth and resistance in response to temperature changes.

MYB transcription factors act as key regulators and affect plant growth and resistance by regulating target genes related to substance synthesis and metabolism ${ }^{21}$. More recent studies in Arabidopsis have shown that overexpression of AtHY5 and AtMYB12 enhanced flavonol accumulation under low-temperature conditions and promoted plant resistance to freezing conditions ${ }^{22}$. In apple plants, MdMYB308L could interact with MdbHLH33 and undergo MdMIEL1-mediated protein degradation to regulate cold tolerance ${ }^{23}$. In maize, sorghum and rice, MYB31 and MYB42 also promoted the expression of the key genes $4 C L 2, F 5 H$, and CSE in the lignin biosynthesis pathway, thus altering lignin production and promoting plant growth ${ }^{24}$. The R2R3-MYB transcription factor MYB15 could accelerate lignification and thereby affect plant growth and resistance in energy-related and agricultural crops by regulating MYB-responsive elements in genes involved in secondary wall biosynthesis ${ }^{25}$. Therefore, investigating the involvement of MYB transcription factors in the response to temperature changes by regulating substance synthesis in apple would be interesting.

In this study, we overexpressed McMYB4 in Malus domestica "Golden Delicious" apple tissue culture seedlings. The results showed that flavonol and lignin accumulation in McMYB4-overexpression (OE) lines is correlated with high McMYB4 expression under different temperature conditions. $\mathrm{Y} 1 \mathrm{H}$ assays and EMSAs revealed that McMYB4 not only targets the promoters of the flavonol biosynthesis genes $C H S$ and $F L S$ but also binds to the promoters of the lignin biosynthesis genes $C A D$ and $F 5 H$ to promote more flavonol and lignin biosynthesis at $28^{\circ} \mathrm{C}$ and $18{ }^{\circ} \mathrm{C}$ than at $23^{\circ} \mathrm{C}$. McMYB4 also promotes IAA and BR signaling pathways by upregulating the expression of $A U X / A R F$ and BRI/BIN. Our findings suggest that McMYB4 improves the temperature adaptation of apple by regulating phenylpropanoid metabolism and hormone signaling.

\section{Results \\ McMYB4 cloning and temperature response analysis}

The novel gene McMYB4, which was identified in the Malus crabapple cultivar "Royalty", contains conserved R2 


\section{(a)}

R2-repeat

MCMYB4: MGRAPCCEKVGLKKGRWTAEEDEILLNYIQANGEGSWRSLPKNAGLLRCGKSCRLRWINYLRA

MdMYB22: MGRAPCCEKVGLKKGRWTAEEDEILLNYIQANGEGSWRSLPKNAGLLRCGKSCRLRWINYLRA

R3-repeat

MCMYB4: DLKRGNISSQEEDIIKLHASLGNRWSLIASQLPGRTDNEIKNYWNSHLSRKIGTFRRPATTTVITTE

MdMYB22: DLKRGNISSQEEDIIIKLHASLGNRWSLIASQLPGRTDNEIKNYWNSHLSRKIGTFRRPATTTVITTE

McMYB4: ISTSSPPAG $\cdots$ ALSSSEITFIKMLII FPDAL

MdMYB22: ISTSVPPAG $\cdots$ ALSSSEINIHQDADHFPDAVISDHQDTELPSSCDQLVPNKVMTTTTTYYGIQ

(b)

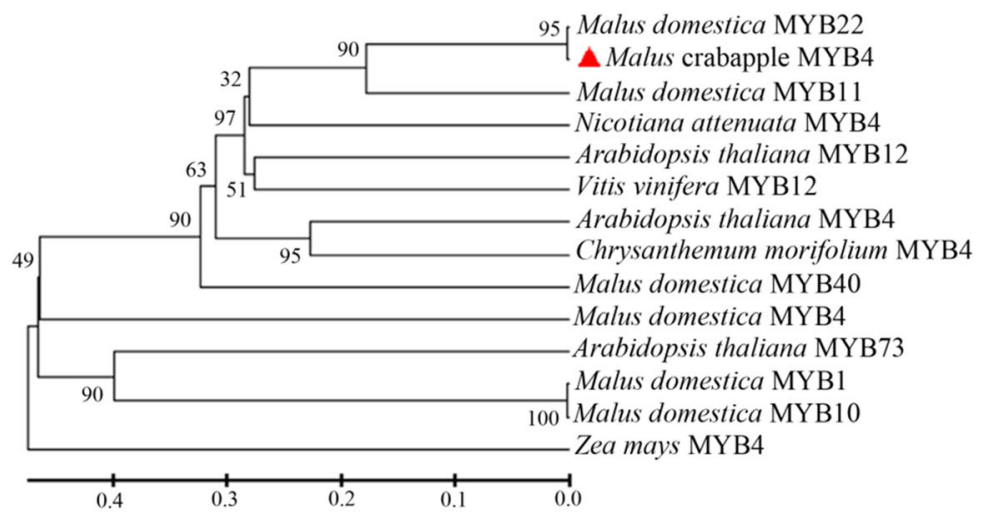

(c)
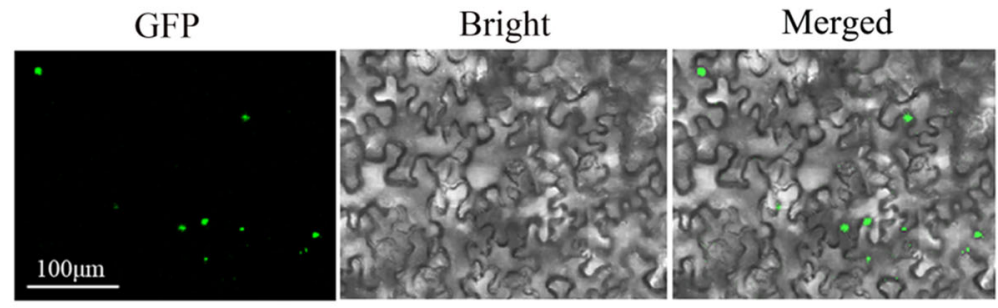

(d)

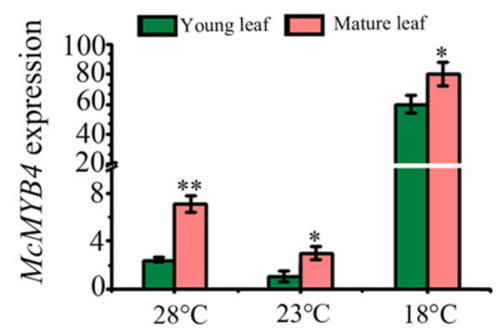

Fig. 1 Bioinformatics and expression analysis of McMYB4. a Amino acid sequence analysis of McMYB4. b Phylogenetic analysis of McMYB4. c Subcellular localization of McMYB4. Bar $=100 \mu \mathrm{m}$. d Expression analysis of MCMYB4. Each bar indicates the mean \pm SD of three repeated experiments $\left({ }^{*} P<0.05,{ }^{* *} P<0.01\right.$, Student's $t$ test)

and R3 DNA-binding domains, and a 33 amino acid deletion was found in the C-terminus of McMYB4 compared with that of $M$. domestica MdMYB22 (Fig. 1a). The phylogenetic tree showed that the similarity between McMYB4 and MdMYB22 was 95\% (Fig. 1b), and a subcellular localization analysis revealed that McMYB4 is located in the nucleus (Fig. 1c).

$M d M Y B 22$ was significantly induced in the "Gala" apple cultivar by low temperature exposure, and its expression also correlated strongly with anthocyanin accumulation ${ }^{21}$. To explore the temperature response of McMYB4 in "Royalty", we cultivated tissue culture seedlings of "Royalty" under $28{ }^{\circ} \mathrm{C}$ and $18{ }^{\circ} \mathrm{C}$ conditions with $23^{\circ} \mathrm{C}$ as the control. qRT-PCR indicated that $28^{\circ} \mathrm{C}$ treatment increased $M c M Y B 4$ expression by 2.4 -fold in young leaves and 2.1 -fold in mature leaves. In addition, $18^{\circ} \mathrm{C}$ treatment increased McMYB4 expression by 60 -fold in young leaves and 21-fold in mature leaves (Fig. 1d). Therefore, 
McMYB4 expression is responsive to temperature changes in Malus crabapple.

\section{McMYB4 promotes flavonol and lignin biosynthesis in response to temperature changes}

To further explore the temperature response of McMYB4 in "Golden Delicious" apple, we transformed the McMYB4 overexpression vector into "Golden Delicious" apple tissue culture seedlings. We selected higherexpressing McMYB4-OE transgenic plants (lines 1, 10, and 12) and "Golden Delicious" apple tissue culture seedlings (used as the WT control) and grew them at $28^{\circ} \mathrm{C}, 18^{\circ} \mathrm{C}$, and $23^{\circ} \mathrm{C}$ (with $23^{\circ} \mathrm{C}$ as the control) for seven days. The results showed that the WT lines wilted, and McMYB4 overexpression alleviated the severity of wilting (S-Fig. 2). At $18{ }^{\circ} \mathrm{C}$, the survival rate of the WT lines was $27 \%$, and that of the McMYB4-OE lines was $67 \%$. The survival rate of the WT and McMYB4-OE lines was $\sim 100 \%$ at $28^{\circ} \mathrm{C}$ (Fig. 2a). qRT-PCR analysis indicated that McMYB4 expression in the McMYB4-OE lines was 1.3 -fold and 4.1 -fold higher at $28^{\circ} \mathrm{C}$ and $18{ }^{\circ} \mathrm{C}$ than at $23^{\circ} \mathrm{C}$, respectively (Fig. 2b). McMYB4 overexpression promoted flavonol and lignin accumulation. HPLC results showed that the flavonol contents in the McMYB4-OE lines grown at $28^{\circ} \mathrm{C}$ and $18^{\circ} \mathrm{C}$ were $493 \mu \mathrm{g} / \mathrm{g}$ and $2715 \mu \mathrm{g} /$ g higher than those at $23^{\circ} \mathrm{C}$, respectively, which were accompanied by upregulated $C H S$ and $F L S$ expression (Fig. 2c, e, g). The lignin content in the McMYB4-OE lines grown at $28^{\circ} \mathrm{C}$ and $18^{\circ} \mathrm{C}$ was increased by $19200 \mu \mathrm{g} / \mathrm{g}$ and $5370 \mu \mathrm{g} / \mathrm{g}$, respectively, compared with that in the McMYB4-OE lines grown at $23^{\circ} \mathrm{C}$, and these increases were accompanied by upregulated $C A D$ and $F 5 H$ expression (Fig. 2d, f, h). Taken together, the results indicate that McMYB4 overexpression enhances the temperature adaptation of apple plants by promoting flavonol and lignin biosynthesis at $28^{\circ} \mathrm{C}$ and $18^{\circ} \mathrm{C}$.

\section{Transcriptome sequencing analysis of MCMYB4 transgenic "Golden Delicious" apple}

We selected McMYB4-OE plants (line 1) and a WT line for transcriptome sequencing analysis. As shown in the Venn plot, 1962 genes were specifically expressed in the McMYB4-OE line, 1038 genes were specifically expressed in the WT line, and 29469 genes were expressed in both the McMYB4-OE and WT lines (Fig. 3a). Differentially expressed gene (DEG) analysis of the McMYB4-OE and WT lines revealed 231 upregulated genes and 131 downregulated genes based on the criteria $\mid \log 2$ (Foldchange) $\mid>1$ and a $q<0.005$ (Fig. 3b). KEGG analysis indicated that the relevant regulatory pathways of the DEGs were mainly associated with secondary metabolite processes, such as phenylpropanoid metabolism, flavonoid biosynthesis, and brassinosteroid biosynthesis pathways (Fig. 3c). Moreover, diphenyl boric acid 2-aminoethyl ester (DPBA) staining also revealed that the McMYB4-OE line contained higher flavonol contents. Microscopy observations of cross-sections of the petioles under UV light revealed that the McMYB4-OE line contained a higher lignin content and more vascular tissue than the WT line (SFig. 1c).

\section{McMYB4 binds to the promoters of CHS, FLS, CAD, and F5H involved in flavonol and lignin biosynthesis}

To further verify the target genes of McMYB4 in the flavonol biosynthesis pathway, we performed $\mathrm{Y} 1 \mathrm{H}$ assays. The results revealed that the $L a c Z$ reporter gene was activated in yeast transformants containing the ADMcMYB4 vector and vectors harboring the promoters of the target genes BD-CHS and BD-FLS (Fig. 4a). EMSA results also showed that McMYB4 binds to the biotinlabeled promoters of CHS and FLS (Fig. 4c, e). Therefore, these results indicate that McMYB4 can bind to the promoters of $C H S$ and $F L S$ to promote flavonol biosynthesis.

$\mathrm{Y} 1 \mathrm{H}$ assays also showed that the $A b A i$ reporter gene was activated in yeast transformants containing the ADMcMYB4 vector and vectors harboring the promoters of the target genes $C A D$ and $F 5 H$ (Fig. 4b), and EMSAs demonstrated that McMYB4 binds to the biotin-labeled promoters of $C A D$ and $F 5 H$ (Fig. 4d, f). Therefore, these results show that McMYB4 can also bind to the promoters of $C A D$ and $F 5 H$ to promote lignin biosynthesis.

\section{MCMYB4 transgenic "Golden Delicious" apple responds to temperature changes by regulating IAA and BR signaling}

To analyze the function of McMYB4 in phytohormone signaling pathways in response to temperature changes, we subsequently evaluated the hormone contents of IAA and $\mathrm{BR}$ in the WT and McMYB4-OE lines. The results showed that McMYB4 overexpression promotes IAA and $\mathrm{BR}$ accumulation. Compared with that at $23^{\circ} \mathrm{C}$ (which served as the control), the IAA content was increased by $14.08 \mu \mathrm{g} / \mathrm{L}$ and $10 \mu \mathrm{g} / \mathrm{L}$ in the $M c M Y B 4-\mathrm{OE}$ lines grown at $28{ }^{\circ} \mathrm{C}$ and $18{ }^{\circ} \mathrm{C}$, respectively, and this increase was accompanied by upregulated $A U X$ and $A R F$ expression (Fig. $5 \mathrm{a}, \mathrm{c}, \mathrm{e}$ ). In addition, the BR content was increased by $14 \mu \mathrm{g} / \mathrm{L}$ and $18 \mu \mathrm{g} / \mathrm{L}$ in the McMYB4-OE lines grown at $28^{\circ} \mathrm{C}$ and $18{ }^{\circ} \mathrm{C}$, respectively, compared with that at $23^{\circ} \mathrm{C}$, and this change was accompanied by upregulated $B R I$ and $B I N$ expression (Fig. 5b, d, f). Moreover, $\mathrm{Y} 1 \mathrm{H}$ assays and EMSAs indicated that McMYB4 mainly activates the promoters of $A U X, A R F, B R I$, and $B I N$ and promotes the IAA and BR signaling pathways (Fig. 6). Consequently, our results suggest that McMYB4 overexpression may enhance IAA and BR accumulation by promoting the expression of genes related to the IAA and $\mathrm{BR}$ signaling pathways in response to $28^{\circ} \mathrm{C}$ and $18^{\circ} \mathrm{C}$ treatments. 
(a)

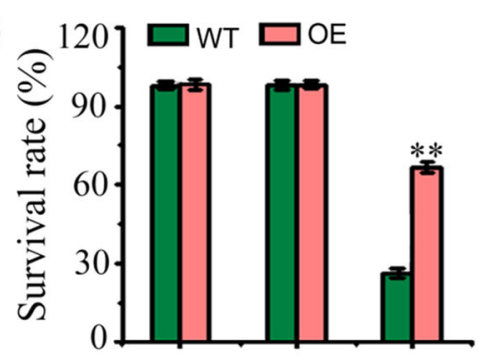

(c)

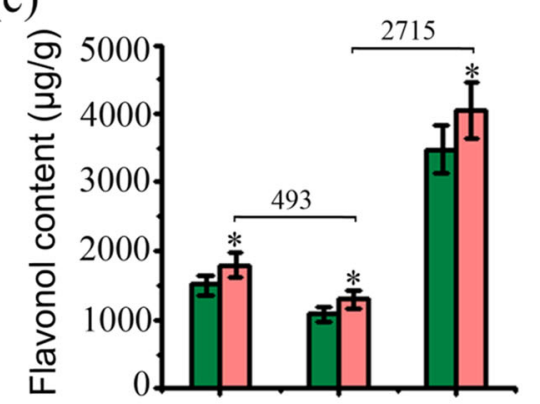

(e)

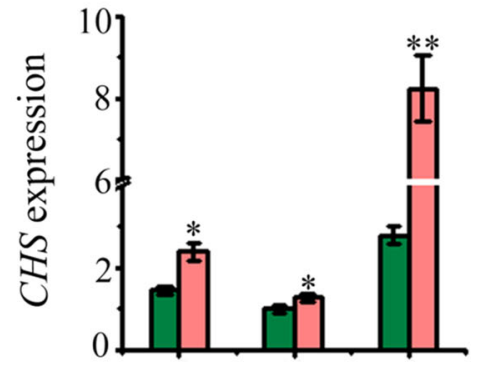

(g)

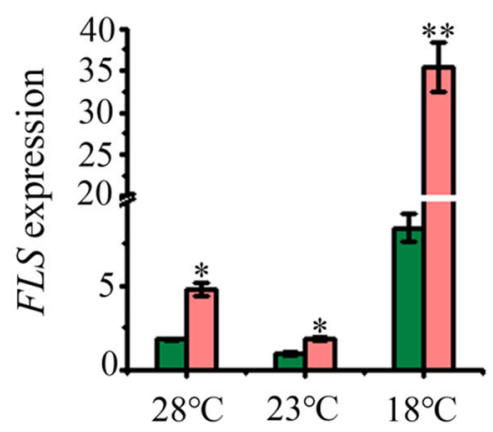

(b)

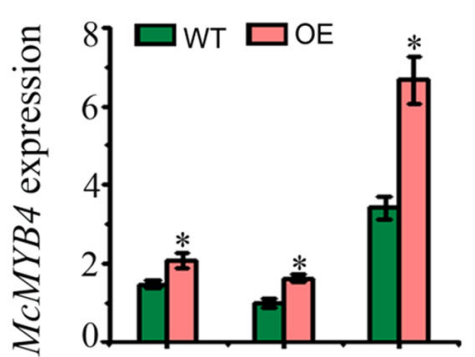

(d)

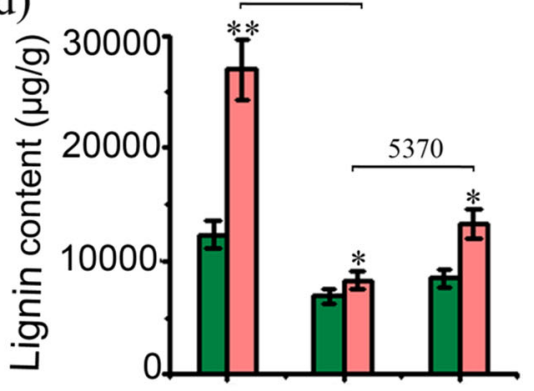

(f)

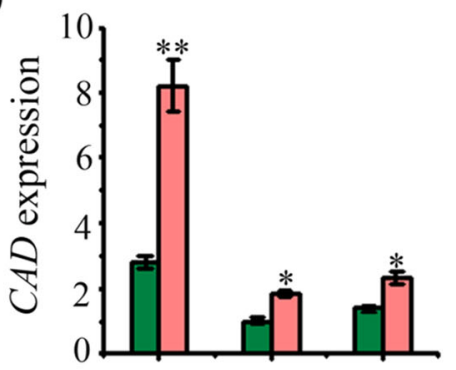

(h)

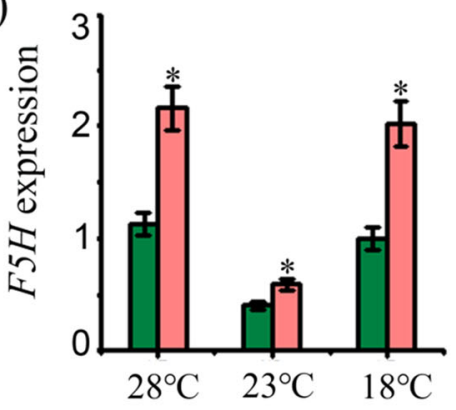

Fig. 2 Flavonol and lignin biosynthesis in the WT and MCMYB4-OE lines in response to temperature changes. a Survival rate of the WT and OE lines during treatment at $28^{\circ} \mathrm{C}$ and $18{ }^{\circ} \mathrm{C} ; 23^{\circ} \mathrm{C}$ served as the control. $\mathbf{b}$ Expression levels of MCMYB4 in the WT and OE lines treated at $28^{\circ} \mathrm{C}$ and $18{ }^{\circ} \mathrm{C} ; 23^{\circ} \mathrm{C}$ served as the control. c , d Flavonol and lignin contents in the WT and OE lines grown at $28^{\circ} \mathrm{C}$ and $18{ }^{\circ} \mathrm{C} ; 23^{\circ} \mathrm{C}$ served as the control. e- $\mathbf{h}$ Expression levels of structural genes in the flavonol and lignin biosynthesis pathways (CHS, CAD, FLS, and F5H) in the WT and OE lines treated at $28^{\circ} \mathrm{C}$ and $18{ }^{\circ} \mathrm{C} ; 23^{\circ} \mathrm{C}$ served as the control. Each bar indicates the mean $\pm S D$ of three repeated experiments $\left(* P<0.05,{ }^{* *} P<0.01\right.$, Student's $t$ test)

MCMYB4 overexpression decreases ROS levels in apple plants under different temperature conditions

ROS are important signaling molecules in plants that play roles in the response to temperature changes. Therefore, we analyzed ROS levels in the WT and McMYB4-OE lines. The leaves of the WT and McMYB4OE lines at $28^{\circ} \mathrm{C}, 23^{\circ} \mathrm{C}$, and $18^{\circ} \mathrm{C}$ were stained with nitro blue tetrazolium (NBT) (dark blue) and diaminobenzidine (DAB) (dark brown). The results showed that the $28^{\circ} \mathrm{C}$ and $18^{\circ} \mathrm{C}$ treatments increased the intensity of $\mathrm{DAB}$ and NBT staining compared with that observed in the $23^{\circ} \mathrm{C}$ treatment (control), and this increased intensity reflects an increase in $\mathrm{O}_{2}{ }^{-}$and $\mathrm{H}_{2} \mathrm{O}_{2}$ accumulation. Compared with those of the WT lines, the leaves of the McMYB4-OE 
(a)

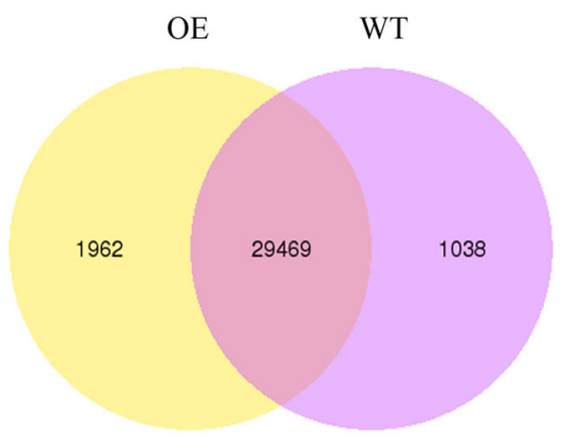

(b)

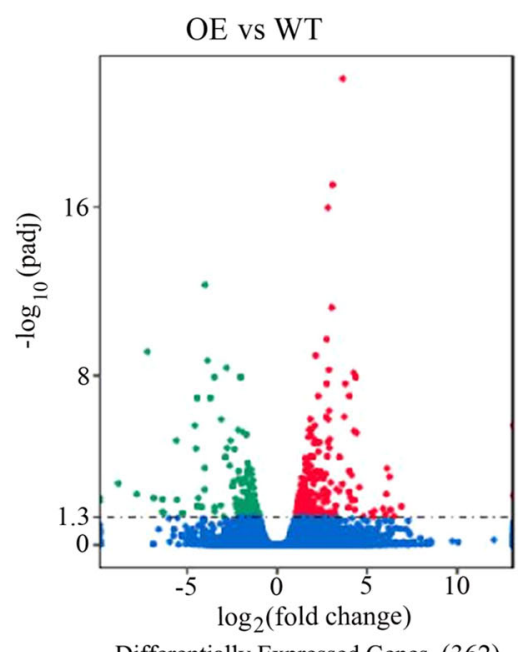

Differentially Expressed Genes (362)

- upregulated: 231

- downregulated: 131

(c)

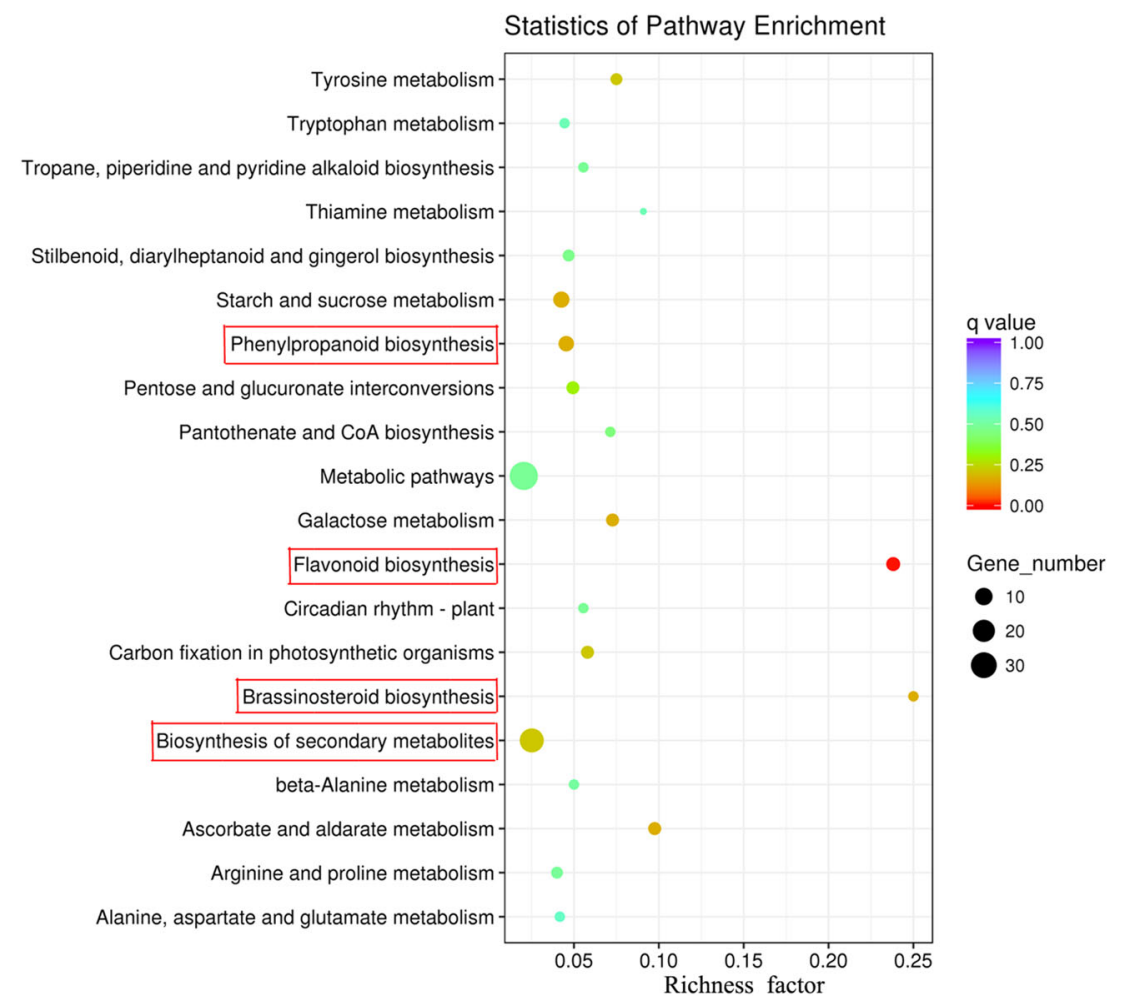

Fig. 3 Transcriptome analysis. a Venn plot analysis of the WT and MCMYB4-OE lines. b DEG volcano plot analysis of the WT and MCMYB4-OE lines. Red indicates upregulation, and green represents downregulation. c KEGG enrichment analysis. The ordinate indicates the pathway name, the abscissa shows the richness factor, the size of the points represents the number of DEGs in the pathway, and the color of the points corresponds to different $q$ value ranges

lines exhibited lower DAB and NBT staining intensities, which reflected lower levels of $\mathrm{O}_{2}^{-}$and $\mathrm{H}_{2} \mathrm{O}_{2}$ accumulation (S-Fig. 3a, b). Therefore, in the McMYB4-OE lines, the $28^{\circ} \mathrm{C}$ and $18{ }^{\circ} \mathrm{C}$ treatments increased the $\mathrm{O}_{2}{ }^{-}$content by $37 \mu \mathrm{mol} / \mathrm{g}$ and $18 \mu \mathrm{mol} / \mathrm{g}$, respectively (S-Fig. 3c), and increased the $\mathrm{H}_{2} \mathrm{O}_{2}$ content by $0.62 \mu \mathrm{mol} / \mathrm{g}$ and $0.29 \mu \mathrm{mol} / \mathrm{g}$, respectively, compared with the levels found with the control treatment $\left(23^{\circ} \mathrm{C}\right.$; S-Fig. $\left.3 \mathrm{~d}\right)$. The 
(a)

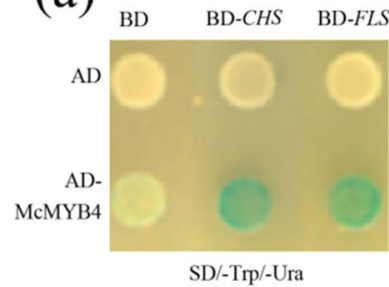

(c)
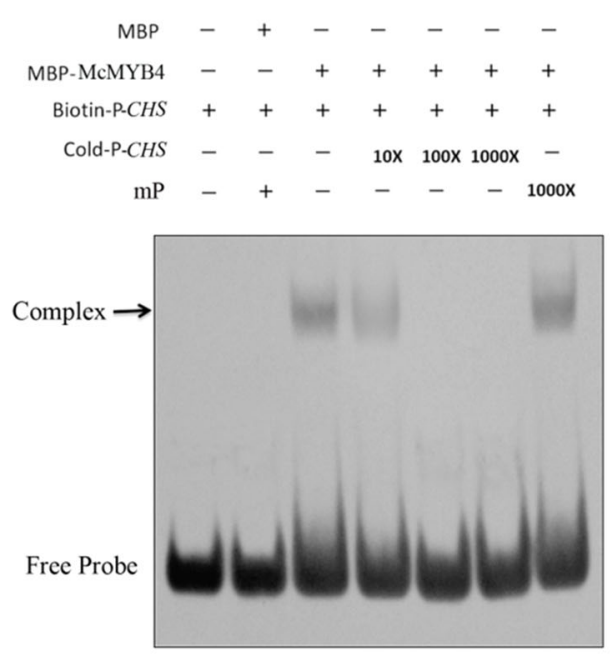

(e)
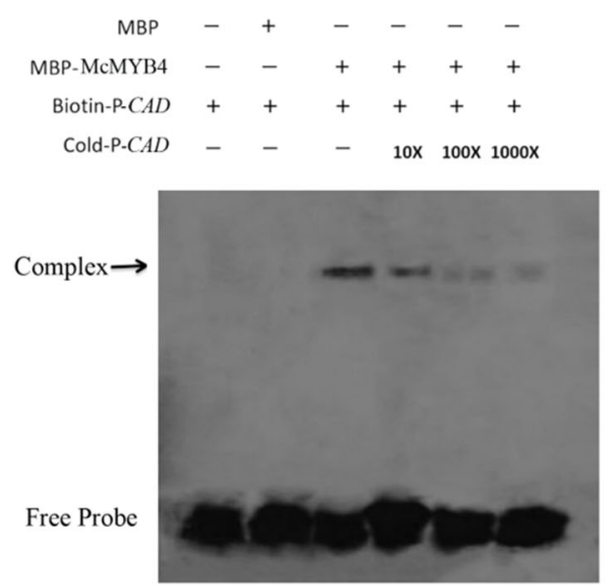

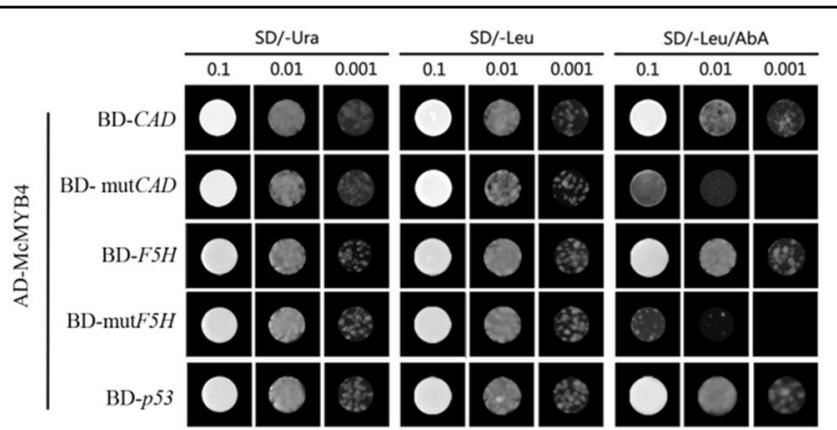

(d)

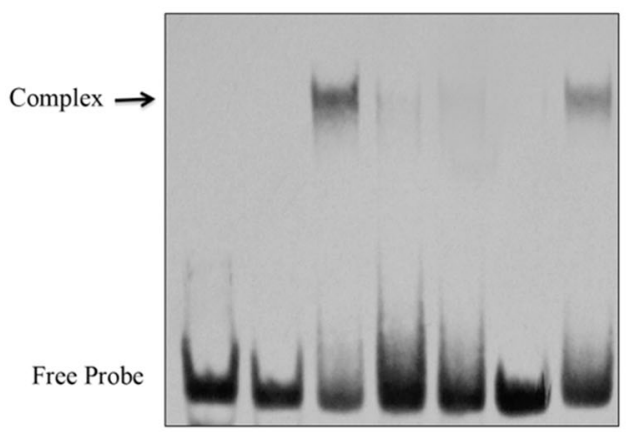

(f)

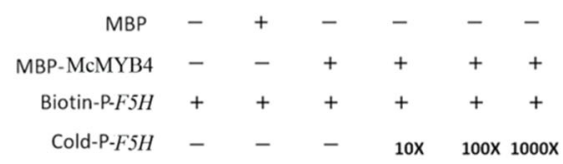

Free Probe

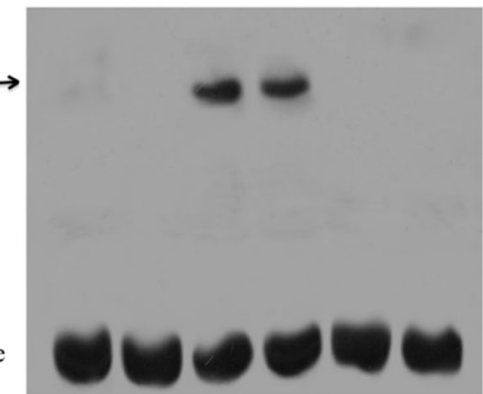

Fig. 4 Analysis of the target genes of McMYB4 in the phenylpropane metabolism pathway. a Binding of McMYB4 to the promoters of the flavonol biosynthesis genes CHS and $F L S$ as measured by $Y 1 \mathrm{H}$ assays. $\mathbf{b}$ Binding of MCMYB4 to the promoters of the lignin biosynthesis genes CAD and $F 5 H$ as determined by $\mathrm{Y} 1 \mathrm{H}$ assays. $\mathbf{c}$, d Binding of MCMYB4 to the promoters of the flavonol biosynthesis genes CHS and FLS as measured by EMSAs. mP: mutant probe. e, $\mathbf{f}$ Binding of MCMYB4 to the promoters of the lignin biosynthesis genes CAD and F5H as determined by EMSAs 
(a)

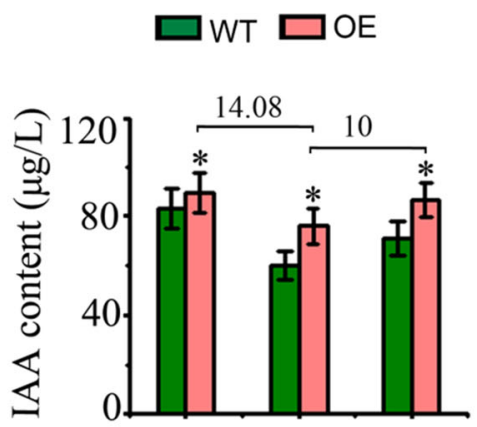

(c)

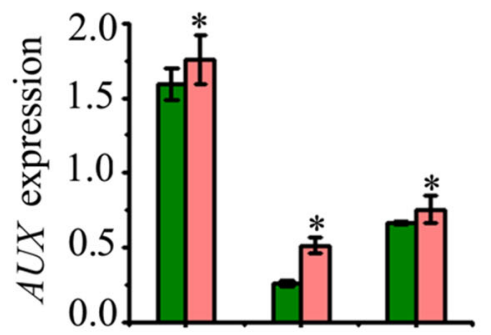

(e)

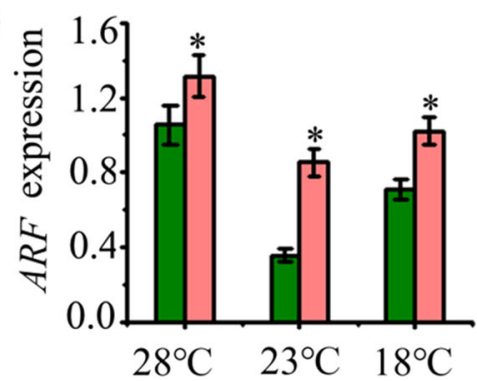

(b)

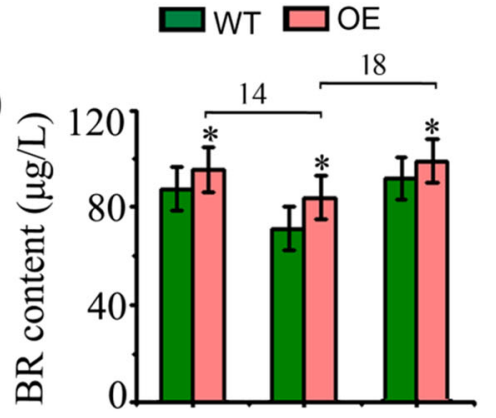

(d)

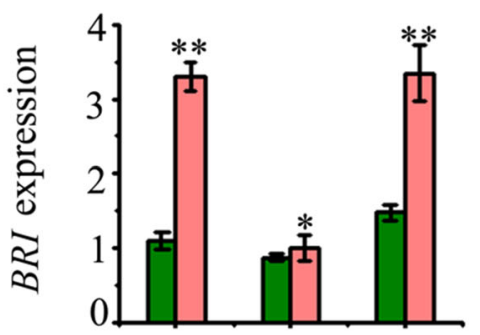

(f)

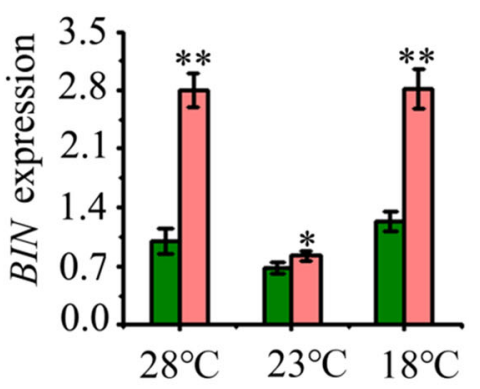

Fig. 5 Analysis of IAA and BR signaling pathways in the WT and McMYB4-OE lines in response to temperature changes. $a, b$ IAA and BR contents in the WT and $\mathrm{OE}$ lines grown at $28^{\circ} \mathrm{C}$ and $18{ }^{\circ} \mathrm{C} ; 23^{\circ} \mathrm{C}$ served as the control. c, e Expression levels of genes in the IAA signaling pathway ( $A U X$ and $A R F$ ) in the plants subjected to the $28^{\circ} \mathrm{C}$ and $18^{\circ} \mathrm{C}$ treatments; $23^{\circ} \mathrm{C}$ served as the control. $\mathbf{d}$, $\mathbf{f}$ Expression levels of genes in the BR signaling pathway $(B R I$ and $B I N)$ with treatment at $28^{\circ} \mathrm{C}$ and $18^{\circ} \mathrm{C} ; 23^{\circ} \mathrm{C}$ served as the control. Each bar indicates the mean $\pm S D$ of three repeated experiments $\left({ }^{*} P<0.05,{ }^{* *} P<0.01\right.$, Student's $t$ test $)$

activities of APX, CAT, and SOD were also higher in the lines grown at $28{ }^{\circ} \mathrm{C}$ and $18{ }^{\circ} \mathrm{C}$ than in those grown at $23^{\circ} \mathrm{C}$ as the control, and the activities of these enzymes in the McMYB4-OE lines were higher than those in the WT lines under the different temperature conditions (S-Fig. $3 \mathrm{e}-\mathrm{g})$. These results indicate that McMYB4 overexpression decreases ROS levels in apple plants by increasing APX, CAT, and SOD activities and thus increases the resistance of plants to $28^{\circ} \mathrm{C}$ and $18^{\circ} \mathrm{C}$.

\section{Discussion}

Temperature changes can regulate apple growth and influence apple production and quality. For example, silencing of the MADS-box gene affected the dormancy and bud break of apple trees in response to chilling temperatures, resulting in an ever-growing or evergreen phenotype and decreased fruit production ${ }^{26}$. In "Fuji" apple, the expression of a B-box transcription factor,
MdCOL4, was promoted by high temperatures. MdCOL4 downregulated $M d M Y B 1$ expression by interacting with MdHY5 under high-temperature conditions, resulting in decreases in apple color intensity and growth ${ }^{27}$. Therefore, understanding the response mechanisms for adapting to temperature changes is crucial for improving apple yields.

\section{McMYB4 promotes flavonol and lignin accumulation in apple plants adapting to temperature changes}

Plants can activate a temperature response by regulating phenylpropanoid metabolism ${ }^{28}$. In tea plants, lowtemperature treatment induced high CsHCT transcription, resulting in the production of chlorogenic acid or acylated flavonol glycosides ${ }^{29}$. In mulberry leaves, low temperatures could also promote the expression of the UFGT gene and lead to flavonoid accumulation ${ }^{30}$. However, the function of McMYB4 in flavonol biosynthesis 


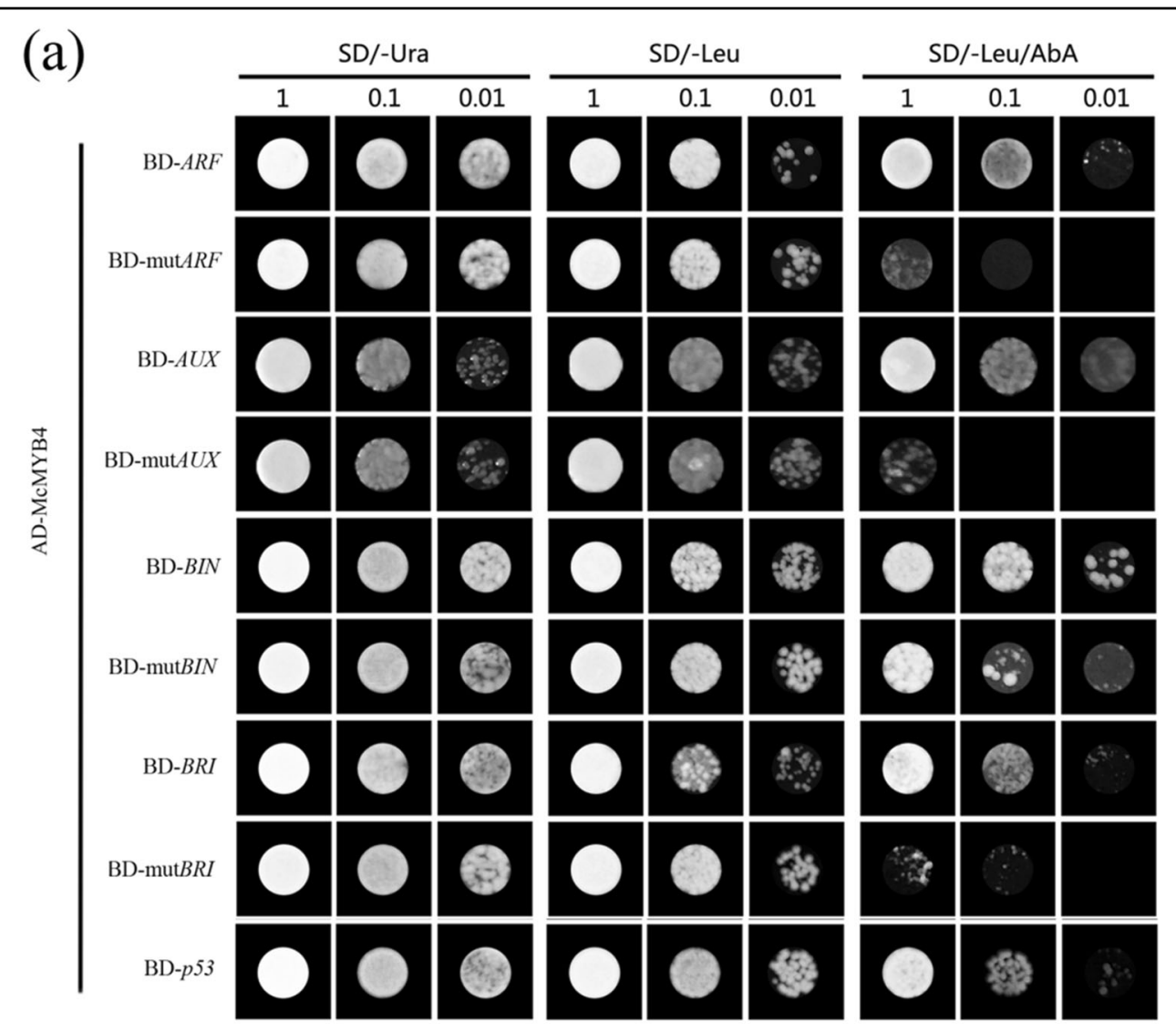

(b)

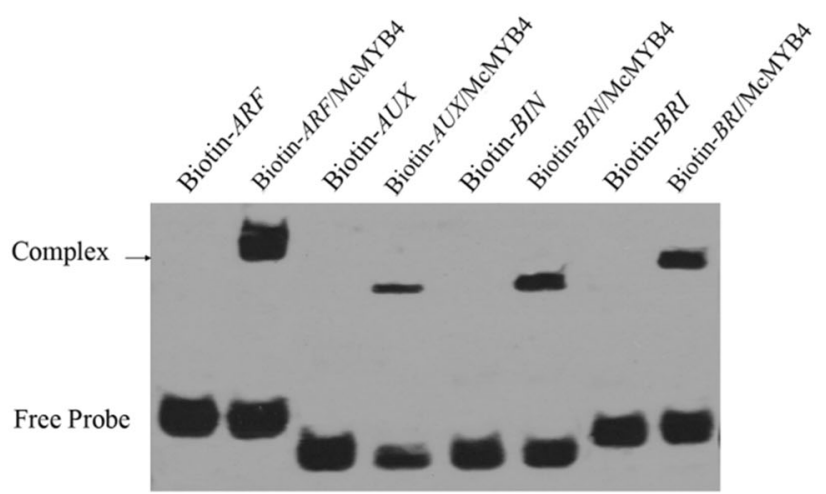

Fig. 6 Analysis of the target genes of McMYB4 in the IAA and BR signaling pathways. a Binding of MCMYB4 to the promoters of the IAA signaling pathway genes $A R F$ and $A U X$ and the BR signaling pathway genes BIN and BRI as determined by $Y 1 H$ assays. $\mathbf{b}$ Binding of McMYB4 to the promoters of the IAA signaling pathway genes ARF and AUX and the BR signaling pathway genes BIN and BRI as determined by EMSAs

pathways in response to temperature changes is unclear. In our study, the expression of CHS and FLS, which have been verified as target genes of McMYB4, was increased in the McMYB4-OE transgenic lines at $28^{\circ} \mathrm{C}$ and $18^{\circ} \mathrm{C}$ (Figs. 2, 4). In addition, MYB TFs also regulate lignin biosynthesis in plant growth and development pro$\operatorname{cesses}^{31}$. In loquat fruit, EjAP2-1 interacted with EjMYB1 and EjMYB2 and transrepressed the promoter of the lignin biosynthesis gene Ej4CL1 to regulate postharvest lignification during low-temperature storage ${ }^{32}$. In maize, ZmMYB31 downregulated the expression of genes involved in monolignol biosynthesis to significantly reduce the lignin content and increase cell wall degradability $^{33}$. In addition, PvMYB4 acts as a master repressor 
of lignin biosynthesis by decreasing the lignin content and S/G lignin ratio in switchgrass plants ${ }^{34}$. However, MdUGT88F1-mediated phloridzin biosynthesis could increase the levels of lignin and cell wall polysaccharides in apple trees, resulting in increases in internode length and stem and adventitious root numbers and improved growth $^{35}$. In our study, the lignin content was increased in the McMYB4-OE lines grown at $28^{\circ} \mathrm{C}$ and $18^{\circ} \mathrm{C}$ compared with the plants grown at $23^{\circ} \mathrm{C}$, and this increase was accompanied by upregulated $C A D$ and $F 5 H$ expression (Figs. 2, 4). Therefore, McMYB4 coordinates the temperature response in apple plants by promoting flavonol and lignin biosynthesis at $18^{\circ} \mathrm{C}$ and $28^{\circ} \mathrm{C}$.

\section{The response of McMYB4 to temperature changes is related to the IAA and BR signaling pathways in apple plants}

Phytohormone signaling can regulate plant resistance and growth in response to temperature changes ${ }^{36}$. In Arabidopsis thaliana, the chromatin-modifying enzyme HDA9 mediated histone deacetylation of a rate-limiting enzyme involved in auxin biosynthesis under high-temperature conditions and promoted auxin accumulation and thermomorphogenesis ${ }^{37}$. Under $28^{\circ} \mathrm{C}$ conditions, the precursor of bioactive GA12 promoted the degradation of DELLAs and induced PIF4-induced upregulation of IAA19/29 expression and hypocotyl elongation in Arabidopsis ${ }^{38}$. Similarly, under $28^{\circ} \mathrm{C}$ conditions, COP1 could regulate BZR in the BR signaling pathway and promote hypocotyl elongation and petiole growth in Arabidopsis ${ }^{39}$. Under low-temperature conditions, RNA-sequencing analysis showed that BR treatment upregulated the expression of several key genes involved in chlorophyll biosynthesis, promoting the photosynthetic capacity and growth of wucai ${ }^{40}$. Moreover, Arabidopsis thaliana SCD1 (stomatal cytokinesis defective 1), a temperature-sensitive allele, could regulate auxin transport and auxin-induced gene expression and affect the gravitropic growth of Arabidopsis in response to different temperatures $\left(25^{\circ} \mathrm{C}\right.$ and $\left.18^{\circ} \mathrm{C}\right)$ by regulating PIN protein trafficking ${ }^{41}$. In our study, McMYB4 overexpression increased not only the IAA and BR contents but also the transcript levels of $A U X I A R F$ and BRI/BIN in the IAA and $B R$ signaling pathways (Figs. 5, 6), suggesting that the response of McMYB4 to temperature changes is related to the IAA and BR signaling pathways in apple plants.

\section{McMYB4 might participate in abiotic resistance and growth by promoting phenylpropanoid biosynthesis and phytohormone signaling in response to temperature changes}

Phenylpropanoids are a large class of secondary metabolites involved in plant resistance and growth in coordination with phytohormone signaling ${ }^{42}$. For example, $p 35 S: F 3 H$ transgenes increased flavonol levels, and flavonol accumulation reduced ROS accumulation, promoting antioxidant activity in tomato under high-temperature conditions ${ }^{43,44}$. In addition, cytokinin could also induce flavonol accumulation in the root transition zone, and a high flavonol content decreased the superoxide radical content, promoting root resistance in Arabidopsis ${ }^{45}$. Moreover, in red mango fruit, RNA-seq analysis suggested that the genes involved in the brassinosteroid signaling pathway were upregulated and increased plant resistance to light ${ }^{46}$. In addition, in brassinosteroid signaling, SERK2, as a BR signaling component, could interact with OsBRI1 to promote early BR signaling and enhance grain size and salt tolerance ${ }^{47}$. Therefore, flavonol biosynthesis and BR signaling have important functions in plant resistance, and during this process, ROS act as plant signals to respond to environmental changes ${ }^{48}$. In watermelon, the $\mathrm{H}_{2} \mathrm{O}_{2}$ content increased and improved adaptation to $4{ }^{\circ} \mathrm{C}$ treatment ${ }^{49}$. In our study, the flavonol levels in the McMYB4-OE lines grown at $28^{\circ} \mathrm{C}$ and $18^{\circ} \mathrm{C}$ were increased by $493 \mu \mathrm{g} / \mathrm{g}$ and $2715 \mu \mathrm{g} / \mathrm{g}$, respectively, compared with those in the control treatment, and the BR contents were increased by $14 \mu \mathrm{g} / \mathrm{L}$ and $18 \mu \mathrm{g} / \mathrm{L}$ in these lines, respectively. The increases in the contents of these metabolites facilitate scavenging of the ROS induced by temperature changes (S-Fig. 3).

As an important component of the cell wall, lignin can affect plant growth. In tea plants, melatonin treatment modified the expression of enzyme genes in the lignin synthesis pathway, increased the lignin content, and promoted plant growth and development ${ }^{50}$. Similarly, in plant growth and development processes, auxin can also affect cell division and elongation to regulate plant growth. For instance, in Arabidopsis, the auxin transcriptional repressor IAA3 interacted with lightcontrolled PIF transcription factors to regulate hypocotyl growth, and disruption of IAA3 led to an elongated hypocotyl under different light intensity conditions ${ }^{51}$. In our study, McMYB4 also promoted lignin biosynthesis and IAA signaling at $28^{\circ} \mathrm{C}$ and $18^{\circ} \mathrm{C}$. The lignin content in the McMYB4-OE lines grown at $28^{\circ} \mathrm{C}$ and $18^{\circ} \mathrm{C}$ was increased by $19200 \mu \mathrm{g} / \mathrm{g}$ and $5370 \mu \mathrm{g} / \mathrm{g}$, respectively, compared with that found in the plants treated at $23^{\circ} \mathrm{C}$, and the IAA content was increased by $14.08 \mu \mathrm{g} / \mathrm{L}$ and $10 \mu \mathrm{g} / \mathrm{L}$ in these lines, respectively. Taken together, the results demonstrated that McMYB4 upregulates flavonol biosynthesis and brassinolide signaling to improve plant resistance during growth at $18^{\circ} \mathrm{C}$. Moreover, McMYB4 upregulates lignin biosynthesis and auxin signaling and thereby promotes plant growth at $28^{\circ} \mathrm{C}$.

In conclusion, McMYB4 increases flavonol and lignin accumulation by promoting the expression of CHS/FLS and $F 5 H / C A D$ in apple at $18^{\circ} \mathrm{C}$ and $28^{\circ} \mathrm{C}$. Moreover, McMYB4 promotes $A U X / A R F$ and BRI/BIN expression and is associated with IAA and BR signaling. Therefore, McMYB4 plays a role in plant resistance and growth by regulating 


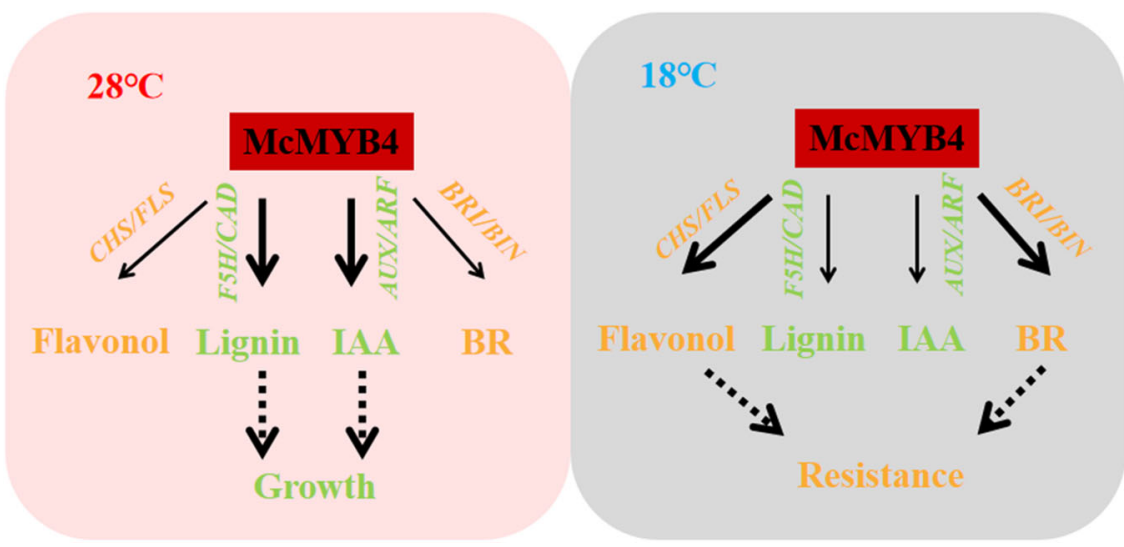

Fig. 7 The regulation model of McMYB4 under different temperature conditions. Yellow: flavonol metabolism and BR signaling pathways; Green: lignin metabolism and IAA signaling pathways; Coarse arrow: strong promotion; Thin arrow: weak promotion; Dotted arrow: proposed MCMYB4 regulated phenylpropane metabolism and hormone signaling to balance growth and resistance homeostasis.

phenylpropanoid metabolism and hormone signaling in response to temperature changes in apple (Fig. 7).

\section{Materials and methods}

Plant materials and growth conditions

Tissue culture seedlings of the Malus crabapple cultivar "Royalty", M. domestica "Golden Delicious" apple (WT), and transgenic "Golden Delicious" apple (lines 1, 10, and 12) were cultivated at the Tissue Culture Center of Beijing University of Agriculture under a $16 / 8$-h photoperiod (1800-2000 lx) and $70 \%$ humidity at $23^{\circ} \mathrm{C}$ for one month. Subsequently, 15 strains were selected from line 1 , line 10 and line 12. Five of 15 strains of the "Royalty" plants, five of 15 strains of the WT plants and five of 15 strains of the McMYB4-OE transgenic plants (lines 1, 10, and 12) were grown in an illumination incubator under a $16 / 8$-h photoperiod (1800-2000 lx) and $70 \%$ humidity at $23^{\circ} \mathrm{C}$ as the control. Five of 15 strains of the "Royalty" plants, five of 15 strains of the WT plants and five of 15 strains of the McMYB4-OE transgenic plants (lines 1, 10, and 12) were grown in an illumination incubator under a $16 / 8$-h photoperiod (1800-2000 lx) and 70\% humidity at $28^{\circ} \mathrm{C}$ (high day temperature). Five of 15 strains of the "Royalty" plants, five of 15 strains of the WT plants and five of 15 strains of the McMYB4-OE transgenic plants (lines 1, 10, and 12) were grown in an illumination incubator under a $16 / 8$-h photoperiod (1800-2000 lx) and 70\% humidity at $18{ }^{\circ} \mathrm{C}$ (low night temperature). After seven days of treatment ${ }^{52}$, all the samples were frozen in liquid nitrogen and stored at $-80^{\circ} \mathrm{C}$ for follow-up assays.

\section{qRT-PCR analysis}

Total RNA was extracted from the leaves in liquid nitrogen under RNase-free conditions via guanidine thiocyanate solution. qRT-PCR was then performed using SYBR $^{\circledR}$ Premix Ex Taq ${ }^{\text {TM }}$ II (Perfect Real Time) (Takara) and a CFX96 ${ }^{\mathrm{TM}}$ Real Time System (Bio-Rad, USA). Malus $18 S$ was used as an internal control, and expression differences were calculated according to the $2^{(-\Delta \Delta \mathrm{Ct})}$ analysis method. The specific primers used for the qRT-PCR analysis are listed in Supplementary Table 1.

\section{Ectopic expression of McMYB4 in "Golden Delicious" apple seedlings}

We cloned McMYB4 from the Malus crabapple cultivar "Royalty", constructed a pH7FWG2-McMYB4-GFP-overexpression vector containing $\mathrm{XbaI}$ and $\mathrm{SacI}$ sites and transformed it into Agrobacterium tumefaciens GV3101. After the A. tumefaciens populations grew to saturation $\left(\mathrm{OD}_{600}=0.8\right)$ in Luria-Bertani media, the culture was centrifuged. The thallus was resuspended in a solution of $10 \mathrm{mM} \mathrm{MgCl} 2,10 \mathrm{mM}$ 2-(N-morpholino) ethanesulfonic acid (MES), and $150 \mathrm{mM}$ acetosyringone and maintained at room temperature for $2 \mathrm{~h}$. The tissue culture seedlings of "Golden Delicious" apple were infected with Agrobacterium containing the corresponding construct using the leaf blade scratch method. Buds were then induced, and positive transgenic buds were transplanted into Murashige and Skoog (MS) media after hygromycin screening and PCR testing. The stable transformation lines $(1,10$, and 12$)$ were selected by qRT-PCR tests (S-Fig. 1a, b). Subculture and expanded propagation of lines 1,10 , and 12 were conducted for follow-up assays.

\section{Subcellular localization of McMYB4}

We transformed the $p H 7 F W G 2-M c M Y B 4-G F P$ vector into Agrobacterium tumefaciens GV3101. The A. tumefaciens populations were cultivated to saturation $\left(\mathrm{OD}_{600}\right.$ $=1.0$ ) in Luria-Bertani media, and the culture was then centrifuged. The thallus was resuspended in a solution of $10 \mathrm{mM} \mathrm{MgCl} 2,10 \mathrm{mM}$ 2-(N-morpholino) ethanesulfonic acid (MES), and $150 \mathrm{mM}$ acetosyringone. Nicotiana 
benthamiana was selected for four weeks and infected via the back of the leaves with a needle-free syringe. The leaves were cultivated for two days and then subjected to laser confocal microscopy (LEICA SP8) observation.

\section{Transcriptome analysis}

We selected tissue culture seedlings of McMYB4-OE transgenic "Golden Delicious" apple (line 1) and "Golden Delicious" apple (WT) for transcriptome sequencing analysis and utilized three sampling times for each plant. The samples were ground immediately in liquid nitrogen, and total RNA was extracted using TRIzol reagent (Invitrogen) according to the manufacturer's protocol. Determination of RNA quantity and purity, the addition of adapters, size selection, and RNA-seq were performed by Novogene (Beijing, China). An RNA-sequencing library was prepared and sequenced using the Illumina $\mathrm{HiSeq}^{\mathrm{TM}}$ platform. Quality control (QC) was performed by analyzing the error rate distribution along reads and the classification of raw reads. DEGseq 1.12.0 was used to analyze the DEGs in each sample based on the criteria $\mid \log 2$ (Fold change) $\mid>1$ and $q<$ 0.005 . Based on the DEG analysis, a KEGG analysis was conducted using KOBAS v2. $0^{52}$.

Measurement of the flavonol, lignin and hormone contents

HPLC was used to measure the flavonol content. Approximately $0.8-1.0 \mathrm{~g}$ (fresh weight) of each sample was placed in a test tube containing $10 \mathrm{~mL}$ of extract solution (methanol:water:formic acid:trifluoroacetic acid $=70: 27: 2: 1)$. The samples were then shaken every $6 \mathrm{~h}$ during incubation at $4{ }^{\circ} \mathrm{C}$ in the dark for $72 \mathrm{~h}$, and the samples were filtered and analyzed by HPLC ${ }^{6}$. The lignin content was measured as described previously and is expressed as $\mathrm{OD}_{280} \mu \mathrm{g} \cdot \mathrm{g}^{-1}(\mathrm{DW})^{34}$. The contents of endogenous IAA/BR were detected by enzyme-linked immunoassay. The data are presented as the means \pm SDs from three repeated experiments.

\section{Microscopic examination}

After staining with DPBA (25 mg of DPBA dissolved in $10 \mathrm{~mL}$ of methyl alcohol) for $30 \mathrm{~min}$, the leaves of tissue culture seedlings of WT and transgenic "Golden Delicious" apple were subjected to microscopy, and the samples were transferred to an objective table for color observations under UV light (Eclipse 80i fluorescence microscope). Cross-sections of the petioles of tissue culture seedlings of WT and transgenic "Golden Delicious" apple were observed under UV light, and images were collected and analyzed using digital imaging software.

\section{$\mathrm{Y} 1 \mathrm{H}$ assays}

The McMYB4 CDS was cloned into a pJG4-5 vector (Clontech) with the galactokinase 1 (GAL1) promoter, which served as an effector construct. The flavonol biosynthesis gene $(C H S / F L S)$ promoter sequences were cloned into a $p L a c Z i$ vector with the $L a c Z$ reporter gene. The vectors were transformed into competent cells of the yeast strain EGY48, which yielded the following yeast strains: $p J G 4-5-M c M Y B 4 / p L a c Z i$-promoter of flavonol regulation genes and $p J G 4-5 / p L a c Z i$-promoter of flavonol regulation genes. The cells were selected on $\mathrm{SD}-\mathrm{Trp} /$-Ura media, and positive colonies were spotted onto glucose plates $(2 \%)$ containing $\mathrm{X}$-gal at $28^{\circ} \mathrm{C}$ for two days to confirm the development of a blue color.

Simultaneously, the $M c M Y B 4$ CDS was also cloned into a $p G A D T 7$ vector ( $p G A D T 7-M c M Y B 4)$, the promoter sequences of the lignin biosynthesis genes $C A D$ and $F 5 H$ and hormone signal genes (AUX/ARF/BRI/BIN) were inserted into a $p A b A i$ vector, and the mutant sequences were also inserted into a $p A b A i$ vector as a negative control. Then, $200 \mathrm{ng}$ of $p G A D T 7-M c M Y B 4$ was added to an Eppendorf (EP) tube, $50 \mu \mathrm{l}$ of $\mathrm{Y} 1 \mathrm{H}$ gold ( $p C A D$-BaitAbAi, pF5H-Bait-AbAi, pAUX-Bait-AbAi, pAUX MutantAbAi, pARF-Bait-AbAi, pARF Mutant-AbAi, pBRI-Bait$A b A i, p B R I$ Mutant-AbAi, pBIN-Bait-AbAi, and $p B I N$ Mutant- $A b A i)$ was added, and $\mathrm{Y} 1 \mathrm{H}$ gold $(p 53-A b A i)$ was used as a positive control. Cells at different concentrations were selected on SD/-Ura, SD/-Leu, and SD/-Leu/500 ng AbA culture plates, and positive colonies grew normally ${ }^{53}$.

\section{EMSAs}

The McMYB4 CDS was cloned into a $p M A L-C 2 X$ expression vector, and the resulting vector was subsequently transformed into Escherichia coli Rosetta (DE3) competent cells. The MBP tag was also cloned in the $p M A L-C 2 X$ vector to purify the recombinant protein. Isopropyl $\beta$-D-1-thiogalactopyranoside (IPTG; $0.3 \mathrm{mM}$ ) was used to induce McMYB4 expression at $170 \mathrm{rpm}$ for $6 \mathrm{~h}$ at $28^{\circ} \mathrm{C}$. The recombinant protein was purified using a One-Step MBP-Tagged Protein Mini-prep Pack (BioLab Co., Ltd., Beijing, China). EMSAs were conducted using the Light Shift ${ }^{\circledR}$ Chemiluminescent EMSA Kit (Thermo Fisher Scientific) according to the manufacturer's protocol with $10 \mu \mathrm{g}$ of purified McMYB4 protein and CHS/FLS/ $C A D / F 5 H$ and $A U X / A R F / B R I / B I N$ probes with biotinlabeled oligonucleotides ${ }^{53}$.

\section{ROS and enzyme activity assays}

Leaf tissues $(0.1 \mathrm{~g})$ were ground to powder in liquid nitrogen and then added to an extraction solution. The $\mathrm{O}_{2}{ }^{-}$and $\mathrm{H}_{2} \mathrm{O}_{2}$ contents and enzyme (APX, CAT, and SOD) activities were measured with kits (Solarbio) according to the manufacturer's instructions. The following formulas were used for calculation: $\mathrm{O}_{2}{ }^{-}(\mathrm{nmol} / \mathrm{g})$ $=148.76 *\left(\mathrm{~A}_{530 \mathrm{~nm}}\right.$ sample- $\mathrm{A}_{530 \mathrm{~nm}}$ control +0.0027$) / \mathrm{W}$, $(\mathrm{W}=0.1 \mathrm{~g}) ; \mathrm{H}_{2} \mathrm{O}_{2}(\mu \mathrm{mol} / \mathrm{g})=\Delta \mathrm{A}_{415} \mathrm{~nm}$ sample $/ \Delta \mathrm{A}_{415 \mathrm{~nm}}$ control / W (W=0.1 g); APX (U/g) = 1.79*[(A3-A4)-(A1A2)] / W, (control: $A 1=A_{290 \mathrm{~nm}} 10 \mathrm{~s}, \mathrm{~A} 2=\mathrm{A}_{290 \mathrm{~nm}} 130 \mathrm{~s}$, 
sample: $\left.\mathrm{A} 3=\mathrm{A}_{290 \mathrm{~nm}} 10 \mathrm{~s}, \mathrm{~A} 4=\mathrm{A}_{290 \mathrm{~nm}} 130 \mathrm{~s}, \mathrm{~W}=0.1 \mathrm{~g}\right)$; CAT $(\mathrm{U} / \mathrm{g})=678^{*}(\mathrm{~A} 1-\mathrm{A} 2) / \mathrm{W}, \quad\left(\mathrm{A} 1=\mathrm{A}_{240 \mathrm{~nm}} 5 \mathrm{~s}, \mathrm{~A} 2=\right.$ $\left.\mathrm{A}_{240 \mathrm{~nm}} \quad 65 \mathrm{~s}, \quad \mathrm{~W}=0.1 \mathrm{~g}\right) ; \quad$ and $\mathrm{SOD} \quad(\mathrm{U} / \mathrm{g})=11.4^{*}$ $\left[\left(\Delta \mathrm{A}_{560 \mathrm{~nm}}\right.\right.$ control- $\Delta \mathrm{A}_{560 \mathrm{~nm}}$ sample $) / \Delta \mathrm{A}_{560 \mathrm{~nm}}$ control* $100 \%] /\left[1-\left(\Delta \mathrm{A}_{560 \mathrm{~nm}}\right.\right.$ control $-\Delta \mathrm{A}_{560 \mathrm{~nm}}$ sample $) /$ $\Delta \mathrm{A}_{560 \mathrm{~nm}}$ control*100\%] / W*10 (30 min, $\left.\mathrm{W}=0.1 \mathrm{~g}\right)$.

\section{Data analysis}

Statistical analyses and figure preparation were performed with OriginPro 8 (Origin Lab Corporation, USA) and Photoshop. The error bars for each symbol indicate the means \pm SDs of three biological replicate reactions. The statistical analyses in the study were performed using Student's $t$ test, where ${ }^{*} 0.01<P<0.05$ and ${ }^{* *} P<0.01$.

\section{Acknowledgements}

We thank the Beijing Collaborative Innovation Center for Eco-Environmental Improvement with Forestry and Fruit Trees and the Beijing Nursery Engineering Research Center for Fruit Crops for providing the experimental resources. This research was funded by the National Natural Science Foundation of China (31772263, 31872081), the Construction of Beijing Science and Technology Innovation and Service Capacity in Top Subjects (CEFFPXM2019_014207_000032), the Scientific Research Improvement Project of Beijing University of Agriculture (GZL2014003) and the Agricultural Science and Technology Project of Beijing Municipal Commission of Rural Affairs (No. 20180202).

\section{Author details}

'Beijing Advanced Innovation Center for Tree Breeding by Molecular Design, Beijing University of Agriculture, Beijing 102206, China. ${ }^{2}$ Beijing Bei Nong Enterprise Management Co. Ltd, Beijing 102206, China. ${ }^{3}$ Plant Science and Technology College, Beijing University of Agriculture, Beijing 102206, China. ${ }^{4}$ Beijing Key Laboratory for Agricultural Application and New Technique, Beijing University of Agriculture, Beijing 102206, China. ${ }^{5}$ College of Forestry, Beijing Forestry University, Beijing 100083, China. ${ }^{6}$ College of Life Science, The Chinese University of Hong Kong, Hong Kong, China

\section{Author contributions}

Y.C.Y. and S.L.Z. designed this study and revised the paper; S.X.H. and Y.F.L. contributed to the data analyses and drafted the paper; Z.P. performed the transcriptome sequencing and analysis; E.Y.W. conducted the plant material culture and treatment; L.K.C. performed the transformation experiments; and S.X.H. collected all the samples and performed the qRT-PCRs, Y1H assays, and EMSAs.

\section{Competing interests}

The authors declare no competing interests.

Supplementary information The online version contains supplementary material available at https://doi.org/10.1038/s41438-021-00620-0.

Received: 31 December 2020 Revised: 6 May 2021 Accepted: 20 May 2021 Published online: 01 August 2021

\section{References}

1. Ding, Y. L., Shi, Y. T. \& Yang, S. H. Molecular regulation of plant responses to environmental temperatures. Mol. Plant. 13, 544-564 (2010).

2. Li, H. et al. MPK3- and MPK6-mediated ICE1 phosphorylation negatively regulates ICE1 stability and freezing tolerance in Arabidopsis. Dev. Cell. 43, 630-642 (2017).

3. $\mathrm{Xu}, \mathrm{Y}$. et al. Natural variations of SLG1 confer high-temperature tolerance in indica rice. Nat. Commun. 11, 5441 (2020).
4. Prinzenberg, A. E., Campos-Dominguez, L., Kruijer, W., Harbinson, J. \& Aarts, M. G. M. Natural variation of photosynthetic efficiency in Arabidopsis thaliana accessions under low temperature conditions. Plant Cell Environ. 43, 2000-2013 (2020)

5. Huo, L. Q. et al. MdATG18a overexpression improves basal thermotolerance in transgenic apple by decreasing damage to chloroplasts. Hortic. Res. 7, 1-15 (2020).

6. Tian, J. et al. McMYB10 regulates coloration via activating McF3'H and later structural genes in ever-red leaf crabapple. Plant Biotechnol. J. 13, 948-961 (2015).

7. Wang, Y. C. et al. Ethylene increases the cold tolerance of apple via the MdERF1B-MdCIbHLH1 regulatory module. Plant J. 105, 1-15 (2021).

8. Ma, D. W. \& Constabel, C. P. MYB repressors as regulators of phenylpropanoid metabolism in plants. Trends Plant Sci. 24, 275-289 (2019).

9. Korn, M., Peterek, S., Mock, H. P., Heyer, A. G. \& Hincha, D. K. Heterosis in the freezing tolerance, and sugar and flavonoid contents of crosses between Arabidopsis thaliana accessions of widely vanying freezing tolerance. Plant Cell Environ. 31, 813-827 (2008).

10. Yin, R. et al. Kaempferol 3-O-rhamnoside-7-O-rhamnoside is an endogenous flavonol inhibitor of polar auxin transport in Arabidopsis shoots. N. Phytol. 201, 466-475 (2014).

11. Yang, C. et al. Lignin metabolism involves Botrytis cinerea BcGs1- induced defense response in tomato. BMC Plant Biol. 18, 103 (2018).

12. Jin, K. et al. Imaging the dynamic deposition of cell wall polymer in xylem and phloem in Populus $\times$ euramericana. Planta 248, 849-858 (2018).

13. Takeda, Y. et al. Down regulation of p-COUMAROYL ESTER 3-HYDROXYLASE in rice leads to altered cell wall structures and improves biomass saccharification. Plant J. 95, 796-811 (2018).

14. Šípošová, K, Kollárová, K, Lišková, D. \& Vivodová, Z. The effects of IBA on the composition of maize root cell walls. J. Plant Physiol. 239, 10-17 (2019).

15. Ployet, R. et al. Long cold exposure induces transcriptional and biochemical remodelling of xylem secondary cell wall in Eucalyptus. Tree Physiol. 38, 409-422 (2018).

16. Artlip, T., McDermaid, A., Ma, Q. \& Wisniewski, M. Differential gene expression in nontransgenic and transgenic "M.26" apple overexpressing a peach CBF gene during the transition from eco-dormancy to bud break. Hortic. Res. 6, 86 (2019).

17. Song, $\mathrm{C}$. et al. Genome-wide identification and expression profiling of the YUCCA gene family in Malus domestica. Sci. Rep. 10, 10866 (2020).

18. Bawa, G. et al. Gibberellins and auxin regulate soybean hypocotyl elongation under low light and high-temperature interaction. Physiol. Plant. 170, 345-356 (2020).

19. Chen, T. et al. TRANSTHYRETIN-LIKE and BYPASS1-LIKE co-regulate growth and cold tolerance in Arabidopsis. BMC Plant Biol. 20, 332 (2020).

20. Zhang, F. et al. Effects of low-temperature stress and brassinolide application on the photosynthesis and leaf structure of tung tree seedlings. Front Plant Sci. 10, 1767 (2020).

21. Song, T. T. et al. Identification of new regulators through transcriptome analysis that regulate anthocyanin biosynthesis in apple leaves at low temperatures. PLOS ONE 14, e0210672 (2019).

22. Bhatia, C., Pandey, A., Gaddam, S. R., Hoecker, U. \& Trivedi, P. K. Low temperature-enhanced flavonol synthesis requires light-associated regulatory components in Arabidopsis thaliana. Plant Cell Physiol. 59, 2099-2112 (2018)

23. An, J. P. et al. An apple MYB transcription factor regulates cold tolerance and anthocyanin accumulation and undergoes MIEL1-mediated degradation. Plant Biotechnol. J. 18, 337-353 (2020).

24. Rao, X. \& Dixon, R. A. Current models for transcriptional regulation of secondary cell wall biosynthesis in Grasses. Front Plant Sci. 9, 399 (2018).

25. Chezem, W. R., Memon, A., Li, F. S., Weng, J. K. \& Clay, N. K. SG2-type R2R3-MYB transcription factor MYB15 controls defense-induced lignification and basal immunity in Arabidopsis. Plant Cell 29, 1907-1926 (2017)

26. Moser, M. et al. MdDAM1The MADS-Box gene controls growth cessation and bud dormancy in Apple. Front Plant Sci. 11, 1003 (2020).

27. Fang, H. et al. MdCOL 4 interaction mediates crosstalk between UV-B and high temperature to control fruit coloration in Apple. Plant Cell Physiol. 60, 1055-1066 (2019).

28. Pastore, C. et al. Whole plant temperature manipulation affects flavonoid metabolism and the transcriptome of grapevine berries. Front Plant Sci. 8, 929 (2017). 
29. Sun, C. H., Yang, C. Y. \& Tzen, J. T. C. Camellia sinensis molecular identification and characterization of hydroxycinnamoyl transferase in tea plants (L.). Int J. Mol. Sci. 19, 3938 (2018).

30. Yu, X. et al. Accumulation of flavonoid glycosides and UFGT gene expression in mulberry leaves (Morus alba L.) before and after Frost. Chem. Biodivers. 14, 1-8 (2017).

31. $\mathrm{Xu}, \mathrm{Q}$. et al. Activator- and repressor-type MYB transcription factors are involved in chilling injury induced flesh lignification in loquat via their interactions with the phenylpropanoid pathway. J. Exp. Bot. 65, 4349-4359 (2014).

32. Zeng, J. K. et al. EjAP2-1, an AP2/ERF gene, is a novel regulator of fruit lignification induced by chilling injury, via interaction with EjMYB transcription factors. Plant Biotechnol. J. 13, 1325-1334 (2015).

33. Fornalé, S. et al. ZmMYB31 directly represses maize lignin genes and redirects the phenylpropanoid metabolic flux. Plant J. 64, 633-644 (2010).

34. Liu, W. et al. Switchgrass (Panicum virgatum L.) promoters for green tissuespecific expression of the MYB4 transcription factor for reduced-recalcitrance transgenic switchgrass. Biotechnol. Biofuels 11, 122 (2018).

35. Zhou, K. et al. MdUGT88F1-mediated phloridzin biosynthesis regulates Apple development and Valsa canker resistance. Plant Physiol. 180, 2290-2305 (2019).

36. Wang, F. et al. SHHY5 integrates temperature, light, and hormonev signaling to balance plant growth and cold tolerance. Plant Physiol. 179, 749-760 (2019).

37. van der Woude, L. C. et al. Arabidopsis thaliana HISTONE DEACETYLASE 9 stimulates auxin-dependent thermomorphogenesis in by mediating H2A.Z depletion. PNAS 116, 25343-25354 (2019).

38. Lucie, C. et al. Root-derived GA12 contributes to temperatureinduced shoot growth in Arabidopsis. Nat. Plants 5, 1216-1221 (2019).

39. Nieto, C., Luengo, L. M. \& Prat, S. Regulation of COP1 function by Brassinosteroid signaling. Front Plant Sci. 11, 1151 (2020).

40. Zhao, M. et al. Transcriptome analysis reveals a positive effect of brassinosteroids on the photosynthetic capacity of wucai under low temperature. BMC Genomics 20, 810 (2019).
41. Carole, L. G. et al. A conditional mutation in SCD1 reveals linkage between PIN protein trafficking, auxin transport, gravitropism, and lateral root initiation. Front Plant Sci. 11, 910 (2020).

42. Huot, B., Yao, J., Montgomery, B. L. \& He, S. Y. Growth-defense tradeoffs in plants: a balancing act to optimize fitness. Mol. Plant. 7, 1267-1287 (2014).

43. Maloney, G. S., DiNapoli, K. T. \& Muday, G. K. The anthocyanin reduced tomato mutant demonstrates the role of flavonols in tomato lateral root and root hair development. Plant Physiol. 166, 614-631 (2014).

44. Muhlemann, J. K. Younts, T. L. B. \& Muday, G. K. Flavonols control pollen tube growth and integrity by regulating ROS homeostasis during high-temperature stress. PNAS 115, E11188-E11197 (2018).

45. Silva-Navas, J. et al. Flavonols mediate root phototropism and growth through regulation of proliferation-to-differentiation transition. Plant Cell 28, 1372-1387 (2016).

46. Sudheeran, P. K. et al. Induced defense response in red mango fruit against Colletotrichum gloeosporioides. Hortic. Res. 8, 17 (2021).

47. Dong, N. N. et al. Regulation of Brassinosteroid signaling and salt resistance by SERK2 and potential utilization for crop improvement in rice. Front Plant Sci. 11, 621859 (2020).

48. He, H. M. et al. The Arabidopsis mediator complex subunit 8 regulates oxidative stress responses. Plant Cell koab079, 1-64 (2021).

49. $\mathrm{Li}, \mathrm{H}$. et al. Methyl jasmonate mediates melatonin-induced cold tolerance of grafted watermelon plants. Hortic. Res. 8, 57 (2021).

50. Han, M. H. et al. Exogenous melatonin positively regulates lignin biosynthesis in Camellia sinensis. Int J. Biol. Macromol. 179, 485-499 (2021).

51. Xi, Y. L. et al. IAA3-mediated repression of PIF proteins coordinates light and auxin signaling in Arabidopsis. PLoS Genet. 17, e1009384 (2021).

52. Zhao, C. D. et al. Apple TIME FOR COFFEE contributes to freezing tolerance by promoting unsaturation of fatty acids. Plant Sci. 302, 110695 (2021).

53. Ma, W. Y. et al. Melatonin alters the secondary metabolite profile of grape berry skin by promoting VVMYB14-mediated ethylene biosynthesis. Hortic. Res. 8, 43 (2021). 